\title{
An evaluation of parenteral nutrition services for adult in-patients within an inner city teaching hospital
}

\author{
O. Hamer, K. Owen and S. Burden On Behalf Of The Trust Parenteral Nutrition Working Group \\ Manchester Royal Infirmary, Central Manchester Foundation Trust, Manchester M13 9WL, UK
}

Parenteral nutrition (PN) for adult in-patients is predominately co-ordinated by dietitians at Manchester Royal Infirmary (MRI). Support is sought as required from other healthcare professionals within clinical areas including consultants, pharmacists, biochemists and a nutrition specialist nurse. A review of services took place at MRI following the publication recommending that all hospitals should have a nutrition multi-disciplinary team (MDT) involved in $\mathrm{PN}^{(1)}$. The MRI has 25 wards, more than 800 beds and produces an average of 4380 bags of PN per year; an average of 12 bags per day.

A staff questionnaire was devised to ask medical, surgical and nursing staff within the Trust about their views on the provision of PN services for adult in-patients before recommendations were made to the Trust Nutrition Steering Committee about PN services. Questionnaires were sent to each adult clinical ward within the Trust. The sample included all medical and surgical consultants, ward managers, nutrition link nurses, lead nurses and modern matrons. Additional questionnaires were given out on each ward to nursing and medical staff of any grade by the ward dietitian.

There were 292 questionnaires sent out of which, 57 were completed and returned a response rate of $20 \%$. All responses received were included. For the closed questions results were analysed using Microsoft Office Excel ${ }^{\circledR} 2003$ and for the open ended questions thematic analysis was used; this was coded by two individuals for consistency. Thirty four (60\%) responses were from doctors, of which 22 (39\%) were from consultants; $23(40 \%)$ responses were from nursing staff. There were $25(44 \%)$ responses from staff who frequently worked with PN.

Results showed 34 (60\%) respondents indicated patients requiring PN were assessed in a 'timely fashion' and 29 (51\%) of respondents indicated PN was received in a 'timely fashion'. Barriers for 'timely' initiation of PN were identified as: patients not seen or PN not received in a reasonable timeframe; lack of education at ward level; delays in referral; no weekend or out-of-hours service and delays obtaining access.

Respondents were asked to comment on the benefits of a nutrition support team (NST), 12 (20\%) did not respond. The remaining respondents indicated the benefits included: clinical expertise; effective management; education and training. Preference for a mandatory team was indicated by $27(50 \%)$ respondents and $22(42 \%)$ indicated a preference for an advisory team to give guidance as required, $4(8 \%)$ indicated a combination and $4(8 \%)$ did not respond. Respondents were asked if they would like a NST to be set up within their specialty or division as opposed to a Trust-wide team; 26 (46\%) gave positive responses and 14 (25\%) negative responses, 11 (19\%) did not want a local team and six (10\%) did not respond. The most frequently selected healthcare professionals to be included in a NST were a dietitian (54 respondents), nutrition nurse (49 respondents), pharmacist (46 respondents), physician (34 respondents) and biochemist (27 respondents).

This survey has provided clinical staff with the opportunity to comment on current PN services and to contribute to future management. Staff identified clear benefits of a NST and having MDT involvement was seen as enhancing patients' care in the Trust. Further discussions are required locally to decide whether a nutrition MDT is set up within each division or whether a Trust wide nutrition MDT is required.

1. Stewart JAD, Mason DG, Smith N et al. (2010) National Confidential Enquiry into Patient Outcome and Death: A mixed bag: an enquiry into the care of hospital patients receiving parenteral nutrition. London. ISBN 978-0-9560882-3-9. 